\title{
Stability of Packaged Solid Dosage Forms. IV.1) Shelf-life Prediction of Packaged Aspirin Aluminum Tablets under the Influence of Moisture and Heat
}

\author{
Kiyoshi Nakabayashi,* Takuo Tsuchida, and Hiroyuki Mima \\ Central Research Division, Takeda Chemical Industries, Ltd., \\ Jusohonmachi, Yodogawa-ku, Osaka, 532, Japan
}

(Received December 20, 1980)

\begin{abstract}
In order to predict the shelf lives of solid dosage forms whose active ingredients are susceptible to damage by moisture and heat, the stabilities of aspirin aluminum tablets packaged in a press-through pack and a glass bottle were investigated in a storehouse. The degradation was found to follow apparent zero-order kinetics. Predictions of the stabilities of aspirin aluminum tablets in these packages were carried out by an iterative calculation over a time interval through a mathematical model in which the kinetic parameters of stability were combined with the moisture permeabilities of the packages. In this study, for the purpose of improving the reliability of shelf-life prediction, the effects of moisture and heat on aspirin aluminum stability were analyzed by a multiple regression technique on the basis of the Carstensen equation. The variation of the degradation rate constants was estimated to obtain their confidence limits with a certain probability. It was found that there was a good agreement between the observed data and the predicted values of the aspirin aluminum contents in the packaged tablets, and that the confidence limits of the degradation rate constants gave reliable shelf lives with the expected probability.
\end{abstract}

Keywords-shelf-life prediction; aspirin aluminum; tablet; iterative calculation; multiple regression analysis; package; temperature; relative humidity; moisture content; moisture permeability

In a previous paper, ${ }^{11}$ it was reported that the color of a sugar-coated tablet containing ascorbic acid was affected by moisture and heat, and that the color change of the sugar-coated tablets in moisture-semipermeable packages could be predicted by an iterative calculation procedure over a time interval through a mathematical model in which the kinetic parameters of the color change were combined with the moisture permeabilities of the packages. The mathematical model was promising, but each of the estimated kinetic parameters had some uncertainty arising from the variation in the experimental data. Thus, it seemed necessary to take into account the variances of the estimated parameters in order to improve the reliability of predicted shelf lives.

Some workers ${ }^{2)}$ have presented shelf-life predictions by means of linear regression analysis with a certain confidence level. However, it seems that their prediction methods are appropriate only to a drug-package combination which deteriorates through the action of one factor, for example, heat alone. A drug-package combination which is liable to be degraded by moisture and heat often shows a complicated rather than a linear time-course of change in a characteristic of the drug. Karel et al. ${ }^{3}$ estimated the uncertainty of the predicted values by use of the propagation of errors in functions, and they applied the results to shelf-life predictions; the variance described by Karel et al. ${ }^{3}$ ' was different from that estimated by standard statistical techniques.

The purpose of this study was to improve the reliability of shelf-life predictions for a drug-package combination in which the drug characteristics were degraded by moisture and heat. In this paper, the stabilities of aspirin aluminum tablets were studied in a moisturesemipermeable package and a reference glass bottle, and a multiple regression analysis was applied to the kinetic study on the basis of the Carstensen equations. ${ }^{4)}$ The shelf-life predictions 
were carried out by an iterative calculation procedure described in detail before, ${ }^{1 /}$ and the confidence intervals of the shelf lives were obtained on the multiple regression model.

\section{Experimental}

Materials - Plain tablets (140 mg per tablet) having the following active ingredients were prepared by a usual method: aspirin aluminum, $40 \mathrm{mg}$; ethenzamide, $20 \mathrm{mg}$; and caffeine, $5 \mathrm{mg}$ per tablet. The excipient used in the tablet was a mixture of microcrystalline cellulose-carboxymethylcellulose calciumhydroxypropylcellulose $(4: 2: 1)$, with a small amount of magnesium stearate. All chemicals were of J.P. IX grade. A highly moisture-proof type of press-through pack ${ }^{5}$ (PTP) for the tablets was prepared using a packaging machine. The average area and the average thickness of the PTP pocket were $1.5 \mathrm{~cm}^{2}$ and $0.115 \mathrm{~mm}$, respectively. The moisture permeability was reported previously.5) A glass bottle with a gum-lined metal screw cap was used as a reference package.

Measurements of Moisture Content and Moisture Absorption of the Tablet-The experiments were carried out in the manner described before. ${ }^{5}$ )

Assay of Aspirin Aluminum - - Four tablets were crushed into powder, which was dissolved in $100 \mathrm{ml}$ of sodium fluoride solution (freshly prepared from $1 \mathrm{~g}$ of sodium fluoride with $200 \mathrm{ml}$ of $0.2 \mathrm{~N}$ hydrochloric acid and $200 \mathrm{ml}$ of absolute ethanol). The solution was divided into two parts after filtration; one $(2 \mathrm{ml})$ was hydrolyzed with $5 \mathrm{ml}$ of $0.2 \mathrm{~N}$ sodium hydroxide for $15 \mathrm{~min}$ and then $5 \mathrm{ml}$ of $0.2 \mathrm{~N}$ hydrachloric acid was added, while $10 \mathrm{ml}$ of $0.2 \mathrm{~N}$ sodium chloride was added to the other part $(2 \mathrm{ml})$. Ferric ammonium sulfate solution similar to that described by previous workers $\left.{ }^{6,7}\right)$ was added to each of the two solutions to make $50 \mathrm{ml}$, and the absorptions of the hydrolyzed and the unhydrolyzed solutions, i.e., $A_{t}$ and $A_{t^{\prime}}$, respectively, were read at $525 \mathrm{~nm}$. The absorptions of a hydrolyzed solution and an unhydrolyzed solution of standard aspirin, i.e., $A_{\mathrm{s}}$ and $A_{\mathrm{s}^{\prime}}$, respectively, were obtained in the same manner. The aspirin aluminum content could be obtained in the form:

$$
\left(\begin{array}{l}
\text { Aspirin aluminum } \\
\text { weight }(\mathrm{mg})
\end{array}\right)=\left(\begin{array}{l}
\text { Standard aspirin } \\
\text { weight }(\mathrm{mg})
\end{array}\right) \times \frac{A_{\mathrm{t}}-A_{\mathrm{t}^{\prime}}}{A_{\mathrm{s}}-A_{\mathrm{s}^{\prime}}} \times 1.12
$$

where the value of 1.12 is the ratio of the weight of one mole of aspirin aluminum to that of two moles of aspirin. The initial content of aspirin aluminum was found to be $40.0 \mathrm{mg}$ per tablet, and the initial free salicylate was negligibly small. The contents are expressed as residual concentrations, as will be described later.

Determination of the Degradation Rate of Aspirin Aluminum in the Tablet- The tablets were adjusted to four moisture contents $(2.40 \%, 3.02 \%, 3.79 \%$, and $5.00 \%)$ by humidification over different saturated salt solutions. ${ }^{5)}$ The various humidified samples were sealed in aluminum-foil-laminated films, and placed in constant temperature ovens ${ }^{5)}$ at $60^{\circ} \mathrm{C}, 50^{\circ} \mathrm{C}$, or $40^{\circ} \mathrm{C}$. The samples were assayed at suitable time intervals depending on the storage conditions.

Storage Tests on Packaged Tablets-The tablets packaged in the press-through pack were kept for two years in a storehouse, and were assayed periodically. The control tablets in the glass bottles with gumlined metal screw caps were also placed there in order to compare the effect of moisture permeabilities of packages on the aspirin aluminum stability. The temperature and the relative humidity in the storehouse were recorded with an Ohta thermometer and an Ohta hygrometer.

Prediction Calculation_- The prediction calculations were performed on a Nihon Denshi JEC-5 computer using a FORTRAN program on the basis of Chart 1 as will be described later.

\section{Results and Discussion}

\section{Effects of Temperature and Moisture Content on Aspirin Aluminum Degradation}

Figure 1 shows typical plots of the degradation of aspirin aluminum in the tablets which were kept at $50{ }^{\circ} \mathrm{C}$. The degradation was found to follow apparent zero-order kinetics. The rate constants were estimated by the least-squares method. ${ }^{8)}$ Table I summarizes the observed apparent rate constants of the tablets with various moisture contents under three temperature conditions; it is clear that the degradation rate constants were influenced by moisture and heat. These apparent rate constants were analyzed by means of empirical formulae of Carstensen et al., ${ }^{4}$ Eq. 2 to Eq. 4, in order to elucidate their dependence on moisture and heat:

$$
\begin{aligned}
& d C / d t=-k^{\prime} \cdot C^{n} \\
& k^{\prime}=k \cdot m^{\alpha} \text { or } \ln k^{\prime}=\ln k+\alpha \cdot \ln m \\
& k=A \cdot \exp (-B / T) \text { or } \ln k=\ln A-B / T
\end{aligned}
$$




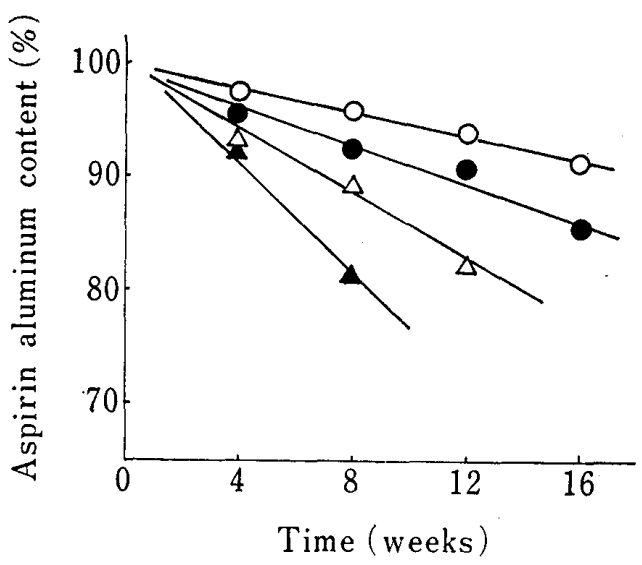

Fig. 1. Zero-order Plots of the Degradation of Aspirin Aluminum in Tablets with Several Moisture Contents at $50{ }^{\circ} \mathrm{C}$

Moisture content: $\bigcirc, 2.40 \%$; $\Delta, 5.00 \%$.
TABle I. Apparent Degradation Rate Constants ${ }^{\alpha)}$ of Aspirin Aluminum in Tablets with Various Moisture Contents at Three Temperatures

\begin{tabular}{cccc}
\hline $\begin{array}{c}\text { Moisture } \\
\text { content }(\%)\end{array}$ & \multicolumn{3}{c}{ Temperature $\left({ }^{\circ} \mathrm{C}\right)$} \\
\hline 20 & 50 & 40 \\
\hline 2.40 & 0.076 & 0.029 & 0.008 \\
3.02 & 0.154 & 0.048 & 0.019 \\
3.79 & 0.241 & 0.083 & 0.032 \\
5.00 & 0.355 & 0.133 & 0.049 \\
\hline
\end{tabular}

a) $\mathrm{mg} \cdot \mathrm{day}^{-1}$.

where $C$ denotes the drug concentration, $m$ the moitsture content, $t$ time, $k$ the rate constant, $k^{\prime}$ the apparent rate constant, $n$ the apparent reaction order, $\alpha$ the interaction order between the drug and the moisture content, $T$ the absolute temperature, while $A$ and $B$ are constants. In this study, $n$ was equal to zero, as described before in this section.

An extrapolation procedure is generally applied for predicting shelf lives, since stability studies are usually carried out under severer conditions than ordinary shelf conditions. The observed degradation rate constants under accelerated conditions generally have some variances arising from the experimental conditions, and thus the rate constants estimated by extrapolation may have larger variances than observed ones. The confidence intervals of the estimated rate constants have a substantial effect in determining shelf lives. ${ }^{2)}$ Multiple regression techniques are useful for analyzing degradation due to many factors. Genton et al., ${ }^{9}$ who applied a multiple regression technique to the analysis of nitrazepam stability, did not refer to the confidence intervals of the degradation rate constants obtained by extrapolation. However, the present authors considered it necessary to adopt degradation rate constants with a confidence interval in order to improve the prediction procedure described before, ${ }^{1,5}$ ) and thus substituted Eq. 4 into Eq. 3 to apply three-parameter regression analysis ${ }^{8)}$ to the data listed in Table I, investigating the $k^{\prime}$-value and the confidence intervals of the individual $k^{\prime}$-values.

The final result was obtained in the form:

$$
\text { ln } k^{\prime}=28.1082+2.1777 \cdot \ln m-10830.8106 / T
$$

Equation 5 indicated that the $\alpha$-value was about two. The individual value of $\ln k^{\prime}$ at absolute temperature, $T$, and moisture content, $m$, lay in the following interval at the $95 \%$ confidence level:8)

$$
\left(\ln k^{\prime}-F \cdot V_{e}^{1 / 2}\right) \mid\left(\ln k^{\prime}+F \cdot V_{e}^{1 / 2}\right)
$$

where $V_{e}$ is expressed in the form:

$$
\begin{aligned}
& V_{e}=1+c_{00}+c_{11} \cdot(\ln m)^{2}+c_{22} \cdot(1 / T)^{2}+2 \cdot c_{01} \cdot(\ln m) \\
& \quad+2 \cdot c_{02} \cdot(1 / T)+2 \cdot c_{12} \cdot(\ln m) \cdot(1 / T) \\
& \left|\begin{array}{lll}
c_{00} c_{01} c_{02} \\
c_{10} c_{11} c_{12} \\
c_{20} c_{21} c_{22}
\end{array}\right|=\left|\begin{array}{lll}
1.3209 \times 10^{2} & -1.4040 & -4.2053 \times 10^{4} \\
-1.4040 & -1.1412 & -1.5736 \times 10^{-3} \\
-4.2053 \times 10^{4} & -1.5736 \times 10^{-3} & 1.3574 \times 10^{7}
\end{array}\right|
\end{aligned}
$$

The values $c_{i j}(i \cdot j=0$ to 2$)$ indicate the inverse matrix of the coefficient matrix for the normal equations, which were used for the multiple regression analysis. The $F$-value is the product of 


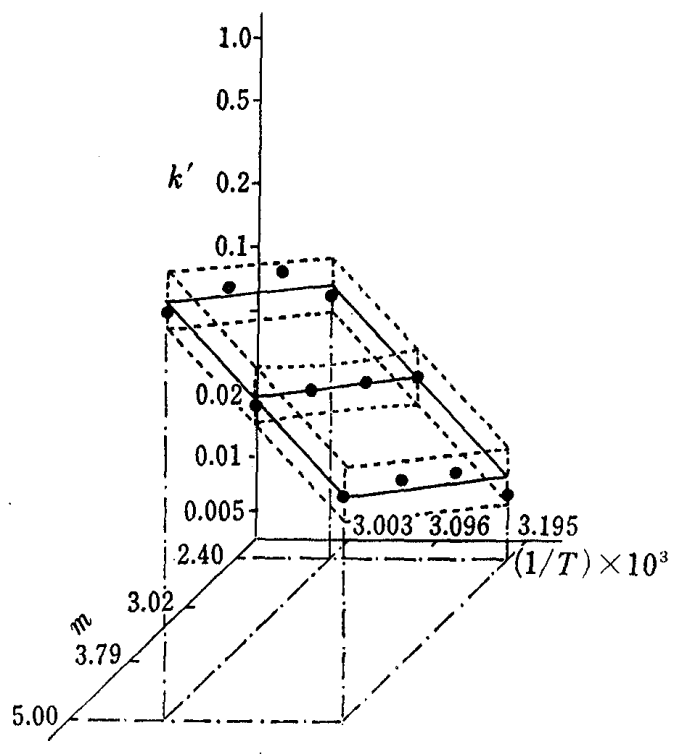

Fig. 2. Variation of the Individual Degradation Rate Constants of Aspirin Aluminum in Tablets

: the observed data; - the most probable value predicted; ......: the lower and upper limits predicted at the confidence level of $95 \%$

Student's " $t$ " and the variance of $\ln k$ ', and it corresponded to 0.2665 in this case. The reason why not the mean value of $k^{\prime}$, but the individual values of $k^{\prime}$ were estimated in this paper is that the shelf life of a product is determined not by an average figure of many lots, but by the individual figures of the lots. Each parameter of Eq. 5 was statistically significant, and the multiple correlation coefficient was so high (0.995) that Eq. 5 was clearly appropriate for expressing the dependence of the stability of aspirin aluminum in the tablets on moisture and heat. However, the value of $k^{\prime}$ had some variances as expressed by Eqs. 6, 7, and 8. For example, the individual value of $k^{\prime}$ at $T=293^{\circ} \mathrm{C}$ and $m=3.50 \%$ was estimated to lie between $1.43 \times 10^{-3} \mathrm{mg}$. day $^{-1}$ and $3.31 \times 10^{-3} \mathrm{mg} \cdot$ day $^{-1}$, while the most probable value was estimated to be $2.18 \times 10^{-3}$ $\mathrm{mg} \cdot$ day $^{-1}$ : the upper limit was 1.5 times the most probable value, while the lower limit was 0.7 times the most probable value. Figure 2 shows a schematic three-dimensional representation of the experimental results and the values estimated by means of Eq. 5 through Eq. 8 .

\section{Moisture Permeabilities of Packaging Materials}

The moisture permeability of the packaging material could be estimated more accurately than the degradation rate, because the permeability measurements were carried out on many samples in each run under temperature conditions involving ordinary conditions in part, as described previously:5) the confidence intervals of permeability constants at the $95 \%$ confidence level were below $10 \%$ of the most probable value under ordinary conditions. The temperature dependence of the permeability constant of the packaging material for the PTP, $P$, studied here was estimated from Eq. $\left.9:{ }^{5}\right)$

$$
P=7.06 \cdot \exp \left(-0.76 \times 10^{3} / T\right)
$$

where the dimensions of the $P$-value are $g \cdot 0.1 \mathrm{~mm} /\left(\mathrm{m}^{2} \cdot \mathrm{cmHg} \cdot\right.$ day $)$. The moisture permeability of the glass bottle was negligibly small and could be disregarded in the predicition calculations.

\section{Moisture Sorption Isotherm}

The moisture sorption isotherm of the tablet could be expressed by a polynomial; under ordinary conditions, the value of the relative humidity, $\mathrm{RH}_{2}$, in equilibrium with the moisture content of the tablet, $m$, could be obtained in the form:

$$
\begin{gathered}
R H_{2}=-88.32+84.35 \cdot m-15.14 \cdot m^{2}+0.96 \cdot m^{3} \\
(2.00 \% \leqq m \leqq 6.50 \%)
\end{gathered}
$$

\section{Storage Tests on Packaged Tablets}

For the purpose of predicting the change in the aspirin aluminum concentration of the packaged tablets during storage, it is necessary to estimate the increase in the moisture content of the tablets due to moisture permeation through the package. Thus, the storage period is divided into many intervals, each of $\Delta t$, and the moisture increase in the tablets through the 
multi-layer overwrapped packaging systems during an interval, $\Delta t$, can be estimated from the relations: ${ }^{1,5)}$

$$
\begin{aligned}
& \Delta m_{j}=\Delta q_{j} / W \\
& \Delta q_{j}=\Delta p_{j} \cdot \Delta t / \Sigma\left\{\left(L_{i} \cdot N_{i}\right) /\left(P_{i . j} \cdot S_{i}\right)\right\} \\
& P_{i, j}=P_{0 . i} \cdot \exp \left\{-E_{i} /\left(R \cdot T_{j}\right)\right\} \\
& \Delta p_{j}=V_{j} \cdot\left(R H_{1 . j}-R H_{2 . j-1}\right) / 100 \\
& V_{j}=V_{0} \cdot \exp \left\{-\Delta H /\left(R \cdot T_{j}\right)\right\} \\
& m_{j}=m_{j-1}+\Delta m_{j} \\
& R H_{2 . j}=\alpha_{0}+\beta_{0} \cdot m_{j}+\gamma_{0} \cdot m_{j}{ }^{2}+\cdots
\end{aligned}
$$

where $j$ denotes one of the time intervals, $i$ a layer of the multilayer overwrapping packaging systems, and $\Delta m_{j}$ the moisture increase (on a dry basis) in the dry tablet weight, $W\left(N_{i}\right.$ tablets in each layer), in the $j$-th interval; $\Delta m_{j}$ is caused by the amount of moisture permeation in the $j$-th interval, $\Delta q_{j}$, through the multi-layer packaging materials, each of which has the surface area, $S_{i}$, the thickness, $L_{i}$, the permeability constant, $P_{i \cdot j}$, and the activation energy of moisture permeation, $E_{i}$, under the condition of $\Delta p_{j}$ vapor-pressure difference across the packaging systems at an ambient absolute temperature, $T_{j}$, for the $j$-th interval. The symbols $m_{j-1}$ and $m_{j}$ are the moisture contents at the ends of the $(j-1)$-th and $j$-th intervals, respectively; $R$ is the gas constant, $V_{j}$ the saturated vapor pressure for the $j$-th interval, and $\Delta H$ the heat of vaporization of water, while $R H_{1 \cdot j}$ is the ambient relative humidity for the $j$-th interval, and $R H_{2 \cdot j-1}$ and $R H_{2 \cdot j}$ are the relative humidities in equilibrium with the moisture contents of the tablets at the ends of the (j-1)-th and $j$-th intervals, respectively. $P_{0 \cdot i}, V_{0}, \alpha_{0}, \beta_{0}, \gamma_{0}$, and the like are constants. In this study, the aspirin aluminum tablets were packaged in the PTP; $i$ was equal to one and Eq. 13 corresponded to Eq. 9. Moreover, Eq. 10 was equivalent to Eq. 17.

\begin{tabular}{|c|c|c|c|c|}
\hline \multirow[b]{2}{*}{ Month } & \multicolumn{2}{|c|}{ 1st year } & \multicolumn{2}{|c|}{ 2nd year } \\
\hline & $\begin{array}{c}\text { Temperature } \\
\left({ }^{\circ} \mathrm{C}\right)\end{array}$ & $\begin{array}{c}\text { Relative } \\
\text { humidity (\%) }\end{array}$ & $\begin{array}{l}\text { Temperature } \\
\left({ }^{\circ} \mathrm{C}\right)\end{array}$ & $\begin{array}{c}\text { Relative } \\
\text { humidity }(\%)\end{array}$ \\
\hline December & 10.5 & 60 & 9.3 & 65 \\
\hline January & 7.0 & 65 & 5.8 & 58 \\
\hline February & 8.4 & 67 & 5.5 & 60 \\
\hline March & 11.0 & 63 & 10.8 & 63 \\
\hline April & 18.1 & 65 & 18.1 & 68 \\
\hline May & 21.6 & 65 & 22.9 & 65 \\
\hline June & 24.8 & 75 & 24.1 & 70 \\
\hline July & 27.2 & 68 & 29.2 & 65 \\
\hline August & 29.1 & 68 & 30.1 & 63 \\
\hline September & 25.8 & 60 & 26.5 & 61 \\
\hline October & 18.8 & 63 & 21.8 & 66 \\
\hline November & 12.0 & 65 & 15.2 & 64 \\
\hline
\end{tabular}

The prediction calculations were reiterated on the basis of the mathematical model described so far over a time interval of 30 days (taken as one month). Table II lists the mean values of the temperature and the relative humidity in each month in the storehouse, which were adopted for the prediction calculations.

TABLE II. Mean Values of Temperature and Relative Humidity in Each Month in the Storehouse (1975-1977)

As described before in this study, the variance of the permeability constants for the packaging materials were smaller than those of the degradation rate constants, and the observed moisture contents of the tablets did not increase infinitely, but remained equivalent to ambient 


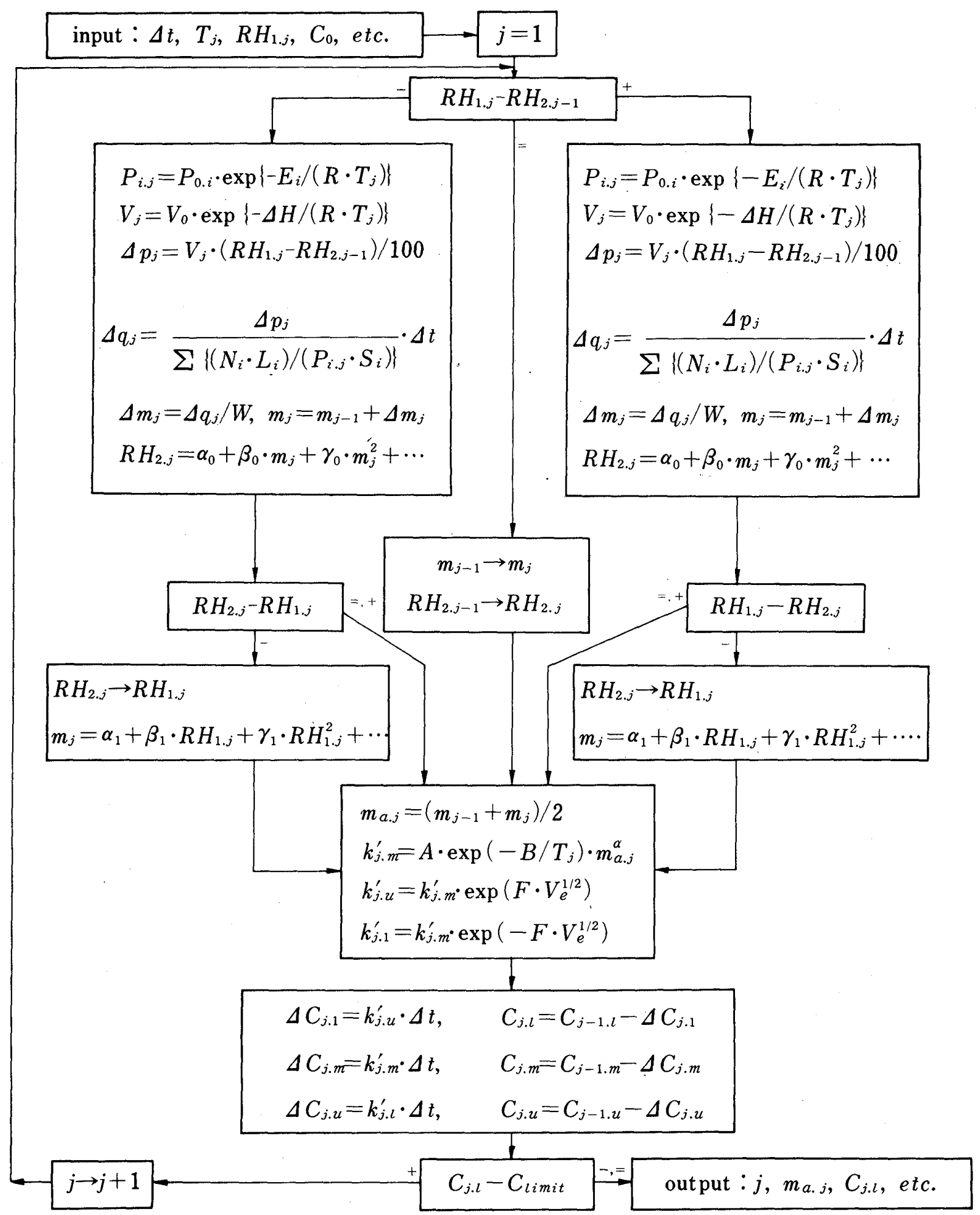

Chart 1. Flow Sheet for the Prediction of Deterioration (Change in C-value) in Tablets Caused by both Moisture and Heat

$C_{0}$ and $C_{l i m i t}$ are the initial $C$-value and the allowable limit, respectively. $C_{j \cdot m}, C_{j \cdot u}$, and $C_{j \cdot l}$ are the most probable value, the upper limit, and the lower limit at the $j$-th interval, respectively. Other notations are given in the text. 
relative humidity. Therefore, it was considered that there was substantial agreement between the predicted moisture contents and the actual data. In addition, Eq. 5 was a multiple regression model derived on the basis of the assumption that the independent variables, i.e., the values of the moisture and the ambient temperature had no errors, and only the dependent variable, i.e., the observed aspirin aluminum content, was subject to error. Thus, the authors neglected the errors in the moisture contents arising from the small variance of the permeability constant of the packaging material on predicting shelf lives, and the average moisture content for each interval was used for the prediction; the average moisture content for a $j$-th interval, $m_{\mathrm{a} \cdot j}$, was obtained from the initial value of the interval, $m_{j-1}$, and the final value of the interval, $m_{j}$, in the form: $:^{5}$

$$
m_{a . j}=\left(m_{j-1}+m_{j}\right) / 2
$$

Since the tablets in this study had a sorption isotherm expressed by Eq. 10, the exclusion of improbable increases or decreases in the moisture content was performed by the use of Eq. 19, as described before: ${ }^{5}$ )

$$
m_{j}=\alpha_{1}+\beta_{1} \cdot R H_{1, j}+\gamma_{1} \cdot R H^{2}{ }_{1 . j}+\cdots
$$

where $\alpha_{1}, \beta_{1}, \gamma_{1}$, and the like are constants.

The degradation of aspirin aluminum in the tablets followed zero-order kinetics as described before, and thus, the decomposed amount, i.e., $\Delta C_{j}$, for a $j$-th interval (30 days), was estimated by use of the value of the apparent rate constant at the $j$-th interval, $k_{j}{ }^{\prime}$, derived from Eq. 5 , into which $T_{j}$ and $m_{a \cdot j}$ were substituted, in the form:

$$
\Delta C_{j}=30 \cdot k_{j}^{\prime}
$$

For $k_{j}{ }^{\prime}$, the most probable value, $k^{\prime}{ }_{j \cdot m}$, the upper limit, $k^{\prime}{ }_{j \cdot u}$, and the lower limit, $k^{\prime}{ }_{j \cdot l}$, were calculated from Eq. 5 through Eq. 8 in order to investigate the variance of the estimated aspirin aluminum content. Summing the value of $\Delta C_{j}$ gives the total estimated amount of decomposed aspirin aluminum in the tablets: Chart 1 shows the flow sheet for the prediction calculation described so far by the use of generalized formulae.

Figure 3 illustrates the time-courses of the observed data and the predicted values of aspirin aluminum in the tablets in both PTP and the glass bottle. The plots of the observed data in the case of PTP showed curves rather than a straight line, though they could be statistically considered linear.

All of the actual data were found to be included in the range of the values predicted through the variances of the $k^{\prime}$-values with confidence limit of $95 \%$. It was found that more degradation occurred in summer, and that the two packages investigated here showed a remarkable difference in effectiveness for preserving aspirin aluminum potency owing to the difference in their moisture permeabilities. If it is assumed that the point where the aspirin aluminum
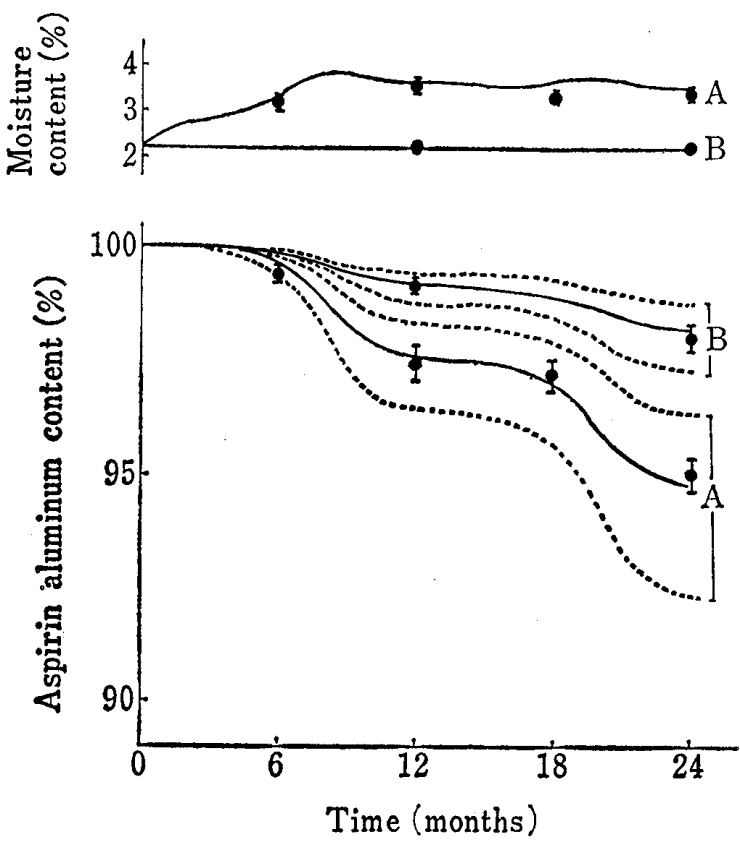

Fig. 3. Comparison of the Observed Data with the Predicted Values Aspirin Aluminum Contents in Packaged Tablets Kept in a Storehouse

A represents tablets packaged in a press-through pack, and $B$ represents tablets in a glass bottle.

$\Phi$ : the observed data; —- the most probable value predicted; -...... the lower and upper limits predicted at the confidence level of $95 \%$. 
content reaches $90 \%$ of the label claim denotes the shelf life, the prediction procedure studied here indicates that the shelf life of the tablets in PTP is 2.5 years at the confidence level of $95 \%$, while that of the tablets in the glass bottle may be more than 5 years at the same confidence level. On the other hand, the conventional prediction technique through linear regression analysis gives a longer shelf life of the tablets in the PTP ( 3.5 years) than that predicted by the procedure in this study. It seems that the iterative calculation technique used in this paper will give reliable shelf lives in terms of taking into account the fluctuations of moisture and temperature during a prolonged period of storage.

It was concluded that degradation rate constants of aspirin aluminum in the tablets could be expressed by a multiple regression model containing the two variables of ambient temperature and moisture content, and that the shelf lives of the tablets in moisture-semipermeable packages could be reliably predicted by an iterative calculation procedure through a mathematical model containing the multiple regression function in part. Thus, the procedure studied here should be helpful in reducing the size and time scale of experiments for forecasting shelf lives of solid dosage forms, and should be useful in package development studies.

\section{References and Notes}

1) Part III: K. Nakabayashi, T. Shimamoto, and H. Mima, Chem. Pharm. Bull.. 28, 1107 (1980).

2) A.J. Woolfe and H.E.C. Worthington, Drug. Development Communication, 1, (3), 185 (1974-1975); J.T. Carstensen and E. Nelson, J. Pharm. Sci., 65, 311 (1976).

3) S. Mizrahi, T.P. Labuza, and M. Karel, J. Food Sci., 35, 799 (1970).

4) J.T. Carstensen, E.S. Aron, D.C. Spera, and J.T. Vance, J. Pharm. Sci., 55, 561 (1966).

5) K. Nakabayashi, T. Shimamoto, and H. Mima, Chem. Pharm. Bull., 28, $1090(1980)$; idem, ibid., 28, 1099 (1980).

6) D. Ribeiro, D. Stevenson, J. Samyn, G. Milosovich, and A.M. Matocks, J. Am. Pharm. Assoc., Sci. Ed., 44, 226 (1955).

7) L.J. Leeson and A.M. Matocks, J. Am. Pharm. Assoc., Sci. Ed., 47, 329 (1958).

8) N.R. Draper and H. Smith, "Applied Regression Analysis," John Wiley and Sons, Inc., New York, 1966, Chapters 1 and 4.

9) D. Genton and U.W. Kesselring, J. Pharm. Sci., 66, 676 (1977). 